\title{
Scoring Model for Prediction of Placenta Accreta Spectrum (PAS) in Early Pregnancy
}

\author{
Original \\ Article \\ Hassan S. Kamel, Diaa Eldeen M. Abd El Aal, Mohammed N. Elammary, \\ Mohammed K. Twfik
}

Department of Obstetrics and Gynecology, Faculty of Medicine, Assiut University, Egypt

\begin{abstract}
Aim: The objective of this prospective cohort study is to create a scoring model for prediction of placenta accreta in early pregnancy by two-dimensional ultrasound and color Doppler.

Materials and Methods: In our study, we investigated 146 Patients at high risk of PAS using two dimensional and color Doppler ultrasound for findings suggestive of placenta accreta in early pregnancy. At time of delivery, diagnosis of placenta accreta was confirmed clinically.

Results: Significant Doppler and 2D ultrasound findings for prediction for PAS in early pregnancy were; low implantation of the gestational sac, presence of placental lacunae and gestational sac or placenta overlapping uterine scar by 2D transvaginal ultrasound and Intraplacental dilated vessels by Doppler ultrasound. A scoring model for prediction of PAS in early pregnancy was created using these significant findings in addition to the number of previous CS deliveries. A cut-off point of 4.5 (out of 7.5 total score value) was used for prediction of cases at high risk of placenta accreta with sensitivity $77 \%$ and specificity $95 \%$.

Conclusion: A standardized risk assessment scoring model based on number of previous cesarean deliveries, 2D- ultrasound and Doppler findings can predict women at highest risk for morbidly adherent placenta in early pregnancy with relatively high specificity.
\end{abstract}

Key Words: Doppler, morbid adherent placenta, placenta accreta, ultrasound

Received: 19 January 2021, Accepted: 16 March 2021

Corresponding Author: Mohammed N. Elammary, Department of Obstetrics and Gynecology, Faculty of Medicine, Assiut University, Egypt, Tel.: +20 01551802097 / +20 01551802097, E-mail: drmnagy@aun.edu.eg

ISSN: 2090-7265, May 2021, Vol.11, No. 2

\section{INTRODUCTION}

Placenta accreta is a substantially life-threatening condition and one of the causes of maternal morbidity and mortality in the world. According to study done in UK, The estimated incidence of placenta accreta/increta/percreta was 1.7 per 10,000 maternities overall and 577 per 10,000 in women with both a previous caesarean delivery and placenta previa ${ }^{[1]}$.

Placenta accreta is considered an increasingly important contributor to repeat cesarean delivery morbidity and clinicians should maintain a high index of suspicion for abnormal placentation and make adequate preparations for patients who need cesarean deliveries ${ }^{[2]}$. Life-threatening obstetric hemorrhage, unplanned Cesarean hysterectomy, and complications related to the abnormal invasion of the placenta into adjacent organs are the catastrophic morbidities associated with placenta accreta $^{[3]}$. The average blood loss at delivery in women with placenta accreta is $3,000-5,000 \mathrm{~mL}^{[4]}$. In addition, $90 \%$ of patients with placenta accreta require blood transfusion and $40 \%$ require more than 10 units of packed red blood cells. Maternal mortality with placenta accreta has been reported to be as high as $7 \%{ }^{[5]}$.

Because placenta accreta can lead to life-threatening blood loss, identification of these high-risk patients would be helpful in management of the pregnancy in addition to enabling the surgeon to be prepared adequately before the time of delivery. Many studies have been done on identification of placenta accreta in the third trimester by two-dimensional (2D) ultrasound and color Doppler ${ }^{[6,7,8]}$. Only few studies have been done on identification of placenta accreta in the early pregnancy using individual ultrasound signs. Sonographic markers of placenta accreta were present as early as first trimester ${ }^{[9,10,11,12]}$. These markers include; low implanted gestational sac, intraplacental lacunae and loss of hypoechogenic retroplacental myometrial zone ${ }^{[13,14]}$.

Early suspicion and diagnosis of placenta accreta in the first trimester provide an ample opportunity to counsel the patient about potential antepartum and intrapartum 
complications, allow for appropriate surveillance, and provide valuable information for a patient to make informed decisions about the remainder of the pregnancy. Additionally, in the event of impending miscarriage or a decision to terminate the pregnancy, an earlier diagnosis has the potential to improve patient safety because physicians and staff can take appropriate steps to ensure the availability of blood products, assemble the proper surgical personnel, and schedule the appropriate surgery and surgical time ${ }^{[15]}$. The main purpose of this study is to predict placenta accreta early in pregnancy through creating scoring model from Ultrasound and color Doppler variables.

\section{PATIENTS AND METHODS}

This is a prospective cohort study conducted in Women Health Hospital, Faculty of Medicine, Assiut University in the period from March 2016 to September 2019. The selected participants had been coming for antenatal care at regular visits. The eligible participants had the following inclusion criteria: Gestational age between 11 weeks and 14 weeks, at least one risk factor for placenta accreta and at least one of the following signs suggestive of placenta accreta (Low implantation of the gestational sac, Presence of placental lacunae, Disruption of placental-myometrial interface, gestational sac or placenta overlapping a uterine scar (CS, myomectomy etc.) by transvaginal ultrasound/ontraplacental dilated vessels or Increased periplacental vascularity by Doppler ultrasound. Exclusion criteria included: pregnant women who refused to $\tau$ articipate or dead fetus.

Sample size calculation was based on a previous longitudinal study by Rahimi-sharpaf et al that reported an incidence of placenta accreta among those with first trimester ultrasound predictors of placenta accreta of $25 \%{ }^{[12]}$. While, the incidence of placenta accreta among those without first trimester ultrasound predictors of placenta accreta was 3.4\%. Using Open EPI application at confidence level $95 \%$ and power $90 \%$, a total sample size of at least 124 cases with 62 in each arm was used.

Gestational age was calculated using Naegle's rule and/or CRL. After informed verbal consent cases were examined by using Medison SONOACE X6 ultrasound device; (for 2D image, transvaginal probe (NEV4-9ES) with the following specifications (Frequency : $6.5[\mathrm{MHz}]$, Number of Elements: 128, Radius of Curvature : 10 [mm] and Field of view : $150\left[^{\circ}\right]$ was used. Transvaginal probe with color Doppler using the optimization settings of the color for umbilical arteries of the index case (gain, pulse repetition frequency and frequency) was used for detection of the placental vasculature). Signs suggestive of placenta accreta by 2D-ultrasound were; low implantation of the gestational sac, presence of placental lacunae (Figure 1), disruption of placental-myometrial interface and gestational sac or placenta overlapping a uterine scar.

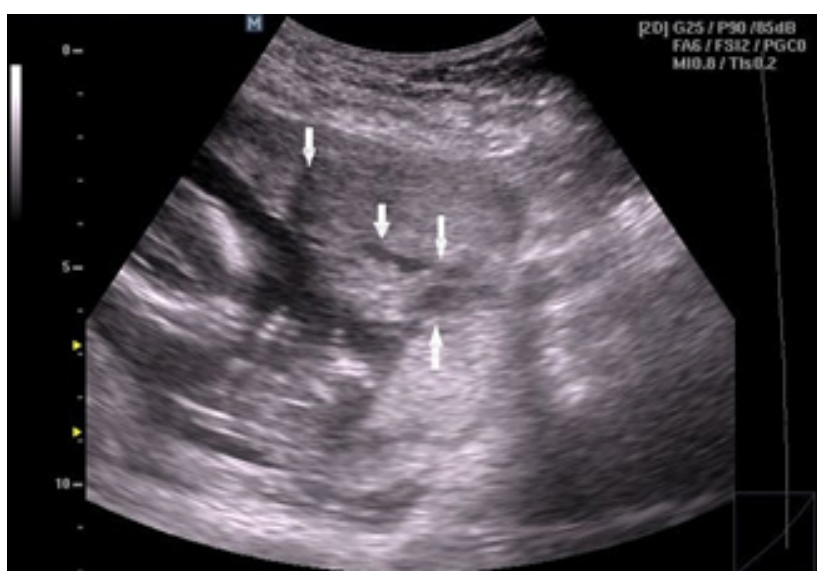

Fig. 1: midsagittal view of 12 weeks pregnancy by $2 \mathrm{D}$ ultrasound showing placental lacunae; anechoic areas within the placenta irregular in shape

Signs suggestive of placenta accreta by color Doppler transvaginal ultrasound were intraplacental dilated vessels (Figure 2) and greatly increased periplacental vascularity (Figure 3). Low implantation of the gestational sac (transvaginal) was determined through a plane defined as mid-sagittal view through the cervical canal showing the isthmus and the lower part of the gestational sac and full length of cervical canal and the inferior edge of the gestational sac measuring $<4 \mathrm{~cm}$ from external cervical os or when the lower placental edge is $<2 \mathrm{~cm}$ from the internal os.

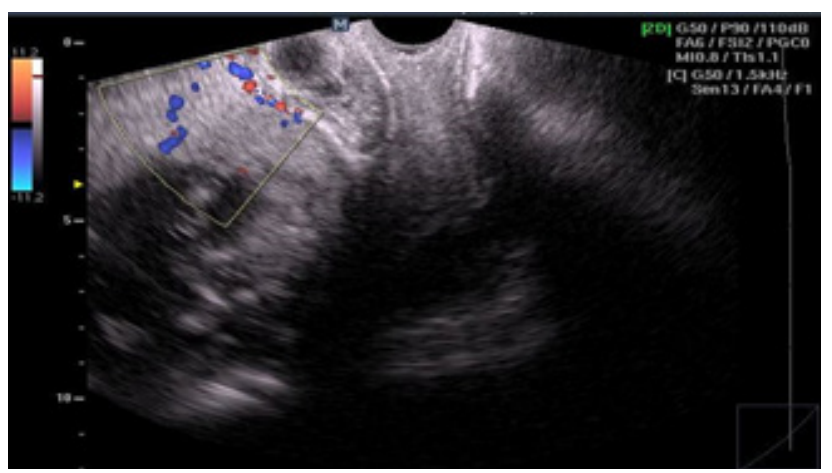

Fig. 2: midsagittal view of 11 weeks pregnancy by 2D ultrasound showing Intraplacental dilated vessels

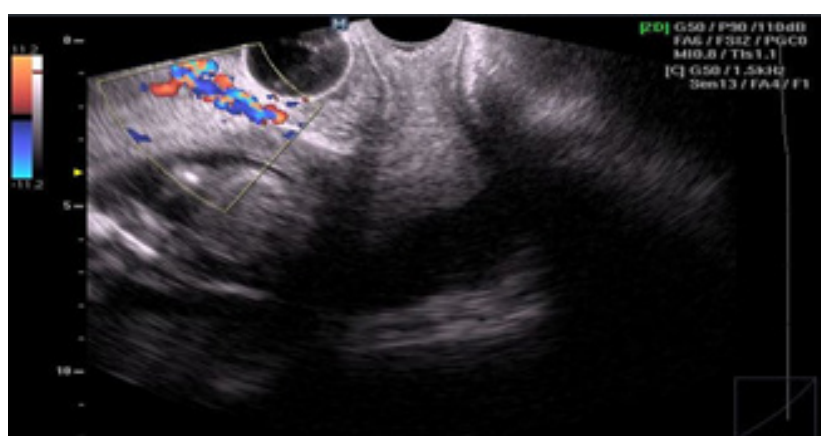

Fig. 3: Midsagittal view of 12 weeks pregnancy by 2D transvaginal ultrasound showing Increased preiplacental vascularity 
Disruption of placental myometrial-interface was defined by presence of irregularity or absence of placentalmyometrial clear interface through panning movement of the probe in the longitudinal plane. Gestational sac or placenta was considered overlapping the scar through a standard plane. This plane was obtained by mid-sagittal view through the cervical canal showing the isthmus and the lower part of the gestational sac. The scar appears as a white thinner area than the surrounding myometrium at the region of the isthmus. The group with at least one of these findings was considered as (group A) and was 73 participants and those without any of these findings was considered as (group B) and were 73 participants.

At time of delivery, the diagnosis of placenta accreta was done clinically by difficult manual, piecemeal removal of placenta if there was no evidence of placental separation 20 minutes after parturition, despite active management in third-stage labor. All data of the participants were collected in a specially designed data collection sheets that include; their contact details and address.

\section{STATISTICAL ANALYSIS}

Data were collected and analyzed using the Statistical Package for Social Science (SPSS Inc., Chicago, version 21). Comparisons between groups were done using Student's t-test to compare the mean values between groups in scale variables. However, $\chi 2$ tests were used to compare the dichotomous and ordinal variables in the groups. For analysis $P<0.05$ was considered significant. The analysis was done for the two groups of the entire cohort at 11 to 14 weeks separate from analysis at 31 to 34 weeks. Subgroup analysis was done for the low sac cohort at 11 to 14 weeks. For creating scoring index, variables which were significant at subgroup analysis of the low sac cohort were given a weighted value for each according to their relative risk. Summing of the score of each case of the low sac cohort and plotted against the placenta accreta state on the receiver operator characteristic curve to detect the optimal criterion value for detection of placenta accreta.

\section{RESULTS}

In the current study, we had studied prospectively a cohort of 146 pregnant women from those attending the antenatal outpatient clinic at Women's Health Hospital, Assiut University, accepting to participate in the study after informed consent and had at least one risk factor for developing Placenta accreta. Five cases failed to be followed (one case from the group $\mathrm{A}$ and 4 cases from the Group B) representing about $3.4 \%$ from all cases. 21 cases became placenta previa and 9 cases have developed placental invasion, two of which had peripartal hysterectomy for uncontrolled bleeding.

Nine cases out of group A developed placenta accreta and 63 cases were without invasion while there were no cases of placenta accreta among group B. Those with clinical evidence of invasion were considered as invasion group while those without clinical evidence of invasion considered as non-invasion group.

Demographic characteristics according to clinical evidence of placental invasion of the entire cohort of the studied population are shown in (Table 1). There were no statistically significant differences between participants who finally had evidence of placental invasion and those without, except for having $\geq 3 \mathrm{CS}$ and history of previous curettage. As regard the number of CS, nearly $90 \%$ of cases with evidence of invasion had at least $3 \mathrm{CS}$ while about $13 \%$ of cases without invasion had at least $3 \mathrm{CS}$ this was highly statistically significant difference $(p=.000)$. Previous curettage had higher incidence among invasion group $(66.7 \%)$ than among the non-invasion group (28\%) which was statistically significant difference $(p=.015)$.

At least one of the 2D ultrasound and Doppler ultrasound parameters of placenta accreta of the $1 \mathrm{st}$ trimester in the entire cohort was detected in each case of the invasion group with a percentage of $100 \%$. In the non-invasion group, only $48 \%$ of cases had at least one of these parameters (i.e., $52 \%$ of cases of the non-invasion group had no any of these parameters) (Table 2). This difference was statistically significant $(p=.002)$. All the studied parameters had shown highly significant prediction of placental invasion apart from Disruption of placental myometrial-interface and greatly increased preiplacental vascularity.

Subgroup analysis was done for the low sac cohort (Table 3). The total number of cases in the low sac cohort was 33 cases. Statistically significant finding for placental invasion were; Placental lacunae, Gestational sac or placenta overlapping the scar, Intraplacental dilated vessels and number of $\mathrm{CS} \geq 3$. As regard placental lacunae, it was present in about $77.8 \%$ among cases of placental invasion and only in $25 \%$ of cases of non-placental invasion and this difference was statistically significant $(p=.006)$. For gestational sac or placenta overlapping the scar, there was significant difference $(P=.000)$ between the group of invasion (88.9\%) and the non-invasion group (20.8\%). Regarding number of CS, presence of at least three CS was associated with statistically significant difference $(P=.001)$ between group with placenta invasion $(88.9 \%)$ and without placental invasion $(25 \%)$.

Variables which were statistically significant in the subgroup analysis from first trimester two dimensional transvaginal (2D) ultrasound parameters and Doppler ultrasound parameters; included gestational sac or placenta overlapping the scar, Intraplacental dilated vessels and Placental lacunae in addition number of $\mathrm{CS} \geq 3$ are presented in (Table 4) according to their relative risk for developing placenta accreta in the cohort of low sac. Relative risk of each parameter and confidence intervals are presented in (Table 4). Of the 9 women with clinical confirmation of placental invasion, $8(89 \%)$ had at least one of these parameters.

For generation of a scoring index for prediction of placenta accreta in the first trimester, each parameter was 
given a weighted value According to the relative risk as presented in (Table 4). Summation of the resulting score for each case and plotting against placenta accreta state in the receiver operating characteristic curve as shown in (Figure 4) was done.

Table1: Demographic characteristics according to clinical evidence of placental invasion of the entire cohort

\begin{tabular}{|c|c|c|c|}
\hline Variable & $\begin{array}{l}\text { Placental invasion, } \\
\mathrm{n}=9\end{array}$ & $\begin{array}{l}\text { No placental invasion, } \\
\mathrm{n}=132\end{array}$ & $P$ value \\
\hline Age $(y)$ mean \pm SD & $29.89 \pm 4$ & $27 \pm 5.2$ & .175 \\
\hline Parity mean $\pm \mathrm{SD}$ & $3.67 \pm 1.4$ & $1.9^{ \pm} 1.4$ & .741 \\
\hline Number of $\mathrm{CS} \geq 3, \mathrm{n}(\%)$ & $8(88.9 \%)$ & $17(12.9 \%)$ & $.000^{*}$ \\
\hline Interval from last pregnancy (y) mean $\pm \mathrm{SD}$ & $2.6 \pm 1.7$ & $2.4 \pm 1.9$ & .656 \\
\hline Previous curettage, $\mathrm{n}(\%)$ & $6(66.7 \%)$ & $37(28 \%)$ & $.015^{*}$ \\
\hline \multirow[b]{3}{*}{$* \mathrm{P}<.05$ significant $\mathrm{y}$; year } & $3(33.3 \%)$ & $41(31.1 \%)$ & \multirow{2}{*}{.887} \\
\hline & $6(66.7 \%)$ & $91(68.9 \%)$ & \\
\hline & \multicolumn{3}{|c|}{$\mathrm{n}$; number of individuals SD; Standard deviation } \\
\hline
\end{tabular}

Table 2: $2 \mathrm{D}$ and Doppler Ultrasound parameters of the $1^{\text {st }}$ trimester in all cases

\begin{tabular}{|c|c|c|c|}
\hline Variable & $\begin{array}{c}\text { Placental invasion, } \\
\mathrm{n}=9\end{array}$ & $\begin{array}{l}\text { No placental invasion, } \\
n=132\end{array}$ & Pvalue \\
\hline Low implantation of the gestational sac & $9(100 \%)$ & $24(18.2 \%)$ & $.000 *$ \\
\hline Placental lacunae & $7(77.8 \%)$ & $29(22 \%)$ & $.000 *$ \\
\hline Disruption of placental myometrial interface & $6(66.7 \%)$ & $67(50.8 \%)$ & .355 \\
\hline Gestational sac or placenta overlapping the scar & $7(77.8 \%)$ & $13(9.8 \%)$ & $.000 *$ \\
\hline Intraplacental dilated vessels & $7(77.8 \%)$ & $26(19.7 \%)$ & $.000 *$ \\
\hline Greatly increased preiplacental vascularity & $5(55.6 \%)$ & $61(46.2 \%)$ & .587 \\
\hline Presence of one or more of $1^{\text {st }}$ trimester predictor signs & $9(100 \%)$ & $63(48 \%)$ & $.002 *$ \\
\hline Values given as number of individuals with the finding $(\%) * P<.05$ significant & & & \\
\hline
\end{tabular}

Table 3: 2D and Doppler Ultrasound parameters of the 1st trimester and number of CS in the low sac cohort versus accreta outcome

\begin{tabular}{lccc}
\hline Variable & Placental invasion, & No placental invasion, & n=24 \\
Placental lacunae & $n=9$ & $6(25 \%)$ & $P$ value \\
Disruption of placental myometrial interface & $7(77.8 \%)$ & $7(29.2 \%)$ & $.006 *$ \\
Gestational sac or placenta overlapping the scar & $6(66.7 \%)$ & $5(20.8 \%)$ & .050 \\
Intraplacental dilated vessels & $8(88.9 \%)$ & $4(16.7 \%)$ & $.000^{*}$ \\
Greatly increased preiplacental vascularity & $7(77.8 \%)$ & $11(45.8 \%)$ & $.001 *$ \\
Number of CS $\geq 3$ & $5(55.6 \%)$ & $6(25 \%)$ & .019 \\
\hline
\end{tabular}

Values given as number of individuals with the finding (\%) $\quad * P<.05$ significant

Table 4: Relative risk estimates confidence intervals (CI) and score of each statistically significant parameter used in the $1^{\text {st }}$ trimester in case of low sac

\begin{tabular}{lccc}
\hline & Parameter & Relative risk & S5\% CI \\
\hline Number of CS $\geq 3$ & 10.8 & $1.5-77$ & 2 \\
Gestational sac or placenta overlapping the scar & 12 & $1.7-87$ & $1.7-28$ \\
Intraplacental dilated vessels & 7 & 1.5 \\
Placental lacunae & 5 & 1.22 \\
\hline
\end{tabular}


Table 5: Sensitivity, specificity, and predictive values for each criterion value of the scoring model.

\begin{tabular}{ccccc}
\hline Criterion & $\begin{array}{c}\text { Sensitivity } \\
(95 \% \mathrm{CI})\end{array}$ & $\begin{array}{c}\text { Specificity } \\
(95 \% \mathrm{CI})\end{array}$ & $\begin{array}{c}\text { PPV } \\
(95 \% \mathrm{CI})\end{array}$ & $\begin{array}{c}\text { NPV } \\
(95 \% \mathrm{CI})\end{array}$ \\
\hline$>1$ & $100(66-100)$ & $45(25-67)$ & $40(20-63)$ & $47-100)$ \\
$>1.5$ & $100(66-100)$ & $58(36-77)$ & $55(29-80)$ & $100(76-100)$ \\
$>2$ & $100(66-100)$ & $70(48-87)$ & $74(42-94)$ & $100(83-100)$ \\
$>2.5$ & $100(66-100)$ & $87(67-97)$ & $79(44-97)$ & $95(78-99)$ \\
$>3$ & $88(51-99)$ & $91(73-99)$ & $87(47-99)$ & $92(74-99)$ \\
$>4.5$ & $77(40-97)$ & $95(78-99)$ & $85(41-99)$ & $88(70-97)$ \\
$>5$ & $66(29-92)$ & $95(78-99)$ & $100(47-100)$ & $85(67-96)$ \\
$>6$ & $55(21-86)$ & $100(85-100)$ & $100(28-100)$ & $80(61-92)$ \\
\hline
\end{tabular}

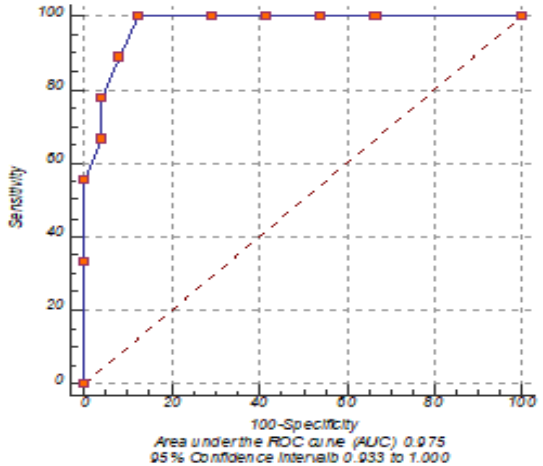

Fig. 4: ROC curve for prediction of placental invasion using ultrasound variables and number of prior cesarean deliveries.

In (Figure 4), a combination of the gestational sac or placenta overlapping the scar, intraplacental dilated vessels and placental lacunae in addition to number $\mathrm{CS} \geq 3$ yielded an area under the receiver operating characteristic curve: $0.975 \quad(95 \% \mathrm{CI} 933$, to 1.000$)$ with $\mathrm{P}$ value $(P=<0.0001)$. The optimal criterion value was $>4.5$ with Sensitivity, specificity, and positive and negative predictive values $77 \%, 95 \%, 87 \%$ and $92 \%$ respectively. So, there is high risk of placenta accreta if there is score $>4.5$. At this cut off point, eight cases from the nine cases with clinical evidence of placenta accreta in our study were detected.

As shown in (Table 5), the summation of the score for a case is presented in criterion column. Each increase in the criterion value is associated with increase in specificity and positive predictive value. Inversely, each increase in the criterion value is associated with decrease in sensitivity and negative predictive value and vice versa. Optimal criterion value was $>4.5$ with best balance between sensitivity, specificity, and positive and negative predictive, $77 \%, 95 \%, 87 \%$ and $92 \%$ respectively.

\section{DISCUSSION}

In our prospective study, we found that all cases with placental invasion had at least one predictor sign. While none of these signs were present in about half of cases without placental invasion: this was significant. These results are consistent with those of a study by Rahimi et al which found that low lying gestational sac and gestational sac or placenta overlapping scar were significantly predictive of placenta accreta. This indicates the ability of these findings for prediction of placenta accreta ${ }^{[12,14]}$.

There was no significant association between maternal age and the occurrence of placental invasion. This finding contrasted with a study done by $\mathrm{Wu}$ et al that found maternal age more than 35 years is a risk factor for placental invasion $^{[5]}$. On the other hand, the presence of previous curettage was associated with more incidence of placenta accreta. This was consistent to a study done by Jauniaux et $a l^{[16]}$. This could be explained by vigorous curettage which destroys parts of the basal endometrium and nitabuch layer leading to placenta accreta evolution.

Our study showed that there were significant association between placental invasion and the following; low sac implantation, placental lacunae, Gestational sac or placenta overlapping the uterine scar and Intraplacental dilated vessels. These significant associations were consistent with many studies ${ }^{[9,17,18]}$. On the other hand, disruption of placental myometrial interface had no significant values between the two groups. This was contradictory to the finding of Ballas et al who reported statistically significant association between disruption of placental myometrialinterface and $\mathrm{PA}^{[10]}$. This difference may be explained by the small number of $1^{\text {st }}$ trimester ultrasound images available for reviewing in his study. Greatly increased preiplacental vascularity was not significantly different between the two groups and this was in contrary to Yang et al who found that there was an association between this finding and $\mathrm{PA}^{[18]}$.

Because the low gestational sac sign was present in all cases that progressed to placenta accreta, so the low sac was considered as a constant factor and we performed a subgroup analysis for the low sac cohort: there were significant association between placental invasion and the following; placental lacunae, gestational sac or placenta overlapping the uterine scar and Intraplacental dilated vessels. These significant associations were consistent with 
many other studies ${ }^{[9,17,18]}$. Furthermore, number of CS $\geq 3$ was significantly associated with the invasion group and thin was coping with Rac et $a l^{[17]}$.

We created a scoring model for prediction of placenta accreta at early pregnancy. Our aim was to standardize prediction of placenta accreta instead of using individual signs for prediction. We constructed the scoring model through using the findings which were statistically significant among the low sac cohort from the first trimester two-dimensional transvaginal (2D) ultrasound parameters and Doppler ultrasound parameters (gestational sac or placenta overlapping the scar, Intraplacental dilated vessels and Placental lacunae) in addition to number of $\mathrm{CS} \geq 3$. Summation of the scores according to presence or absence of these finding was done. The cut off point for prediction of placenta accreta in the score was $>4.5$ with the highest possible specificity (95\%) and associated sensitivity (77\%) indicating that it is a good negative test. The scoring model was highly predictive of placental invasion early in pregnancy among pregnancies at increased risk PA with low sac implantation.

The application of this scoring model for prediction of placenta accreta in early pregnancy can be helpful in identification of pregnant women at high risk of placenta accreta at early pregnancy. For example, if a woman who has had 3 prior cesarean deliveries with low implanted sac is found to have gestational sac or placenta overlapping the scar, and Placental lacunae she would receive 2 points for the prior cesarean deliveries, 1 point for placental lacunae and 2.5 points for gestational sac or placenta overlapping the scar. This would result in a score of 5.5 which is more than 4.5 indicating high possibility of placenta accreta. Conversely, a woman with 2 prior cesarean deliveries $(0$ point), placental lacunae (1 point), Intraplacental dilated vessels (1.5 points), and gestational sac or placenta away from the scar ( 0 points) would have a score of only 2.5 which is less than 4.5 indicating low risk of placenta accreta. According to the availability of local resources and multidisciplinary care, results from our scoring index could thus be used for counseling and assist with referral decisions in case of high possibility of PA.

An important aspect in counseling women at high risk of developing PA (with score more than 4.5) if possible, early termination of pregnancy that may avoid a lot of risks threatening both maternal and fetal life later in pregnancy or in labor. Also, it may be used for counseling and preoperative planning in case of missed abortion at early pregnancy by either raising awareness for morbidly adherent placenta or providing reassurance. It may also play a role in identifying women who may benefit from referral to a tertiary center that has enough blood bank capacity and multidisciplinary services. Also, instead of using each ultrasound variable individually, our model establishes a scoring system for a standardized ultrasound evaluation of all patients at risk for morbidly adherent placenta that can be adopted in high risk women with sac low position.
Up to our knowledge, this is the first predictive scoring index for prediction of placenta accreta in early pregnancy based on prospective study. The previous investigators were using individual ultrasound variables and most of them were based on retrospective $\mathrm{e}^{[10,11,17]}$ studies and few prospective studies ${ }^{[9,10,12,14,17,18]}$.

\section{CONFLICT OF INTEREST}

There are no conflict of interest

\section{REFERENCES}

1. Fitzpatrick KE, Sellers S, Spark P, Kurinczuk JJ, Brocklehurst $\mathrm{P}$, et al. Incidence and risk factors for placenta accreta/increta/percreta in the UK: a national case-control study. PloS one. 2012;7(12):e52893.

2. Creanga AA, Bateman BT, Butwick AJ, Raleigh L, Maeda A, et al. Morbidity associated with cesarean delivery in the United States: is placenta accreta an increasingly important contributor? American Journal of Obstetrics and Gynecology. 2015 9//;213(3):384. e1-384.e11.

3. Silver RM, Landon MB, Rouse DJ, Leveno KJ, Spong $\mathrm{CY}$, et al. Maternal morbidity associated with multiple repeat cesarean deliveries. Obstetrics and gynecology. 2006 Jun;107(6):1226-32.

4. Hudon L, Belfort MA, Broome DR. Diagnosis and management of placenta percreta: a review. Obstetrical \& gynecological survey. 1998 Aug;53(8):509-17.

5. Wu S, Kocherginsky M, Hibbard JU. Abnormal placentation: twenty-year analysis. Am J Obstet Gynecol 2005 May;192(5):1458-61.

6. Yu M, Liu XY, Dai Q, Cui QC, Jin ZY, et al. [Diagnosis and treatment of placenta accreta in the second trimester of pregnancy]. Zhongguo yi xue ke xue yuan xue bao Acta Academiae Medicinae Sinicae. 2010;32(5):501-4.

7. D'ANTONIO F, IACOVELlA C, BHIDE A. Prenatal identification of invasive placentation using ultrasound: systematic review and meta-analysis. Ultrasound Obstet Gynecol 2013;42:509-517.

8. Shih JC, Palacios-Jaraquemada JM, SU YN, Shyu MK, LIN CH, et al. Role of three-dimensional power Doppler in the antenatal diagnosis of placenta accreta: comparison with gray-scale and color Doppler techniques. Ultrasound Obstet Gynecol. 2009;33:193-203. 
9. Ballas J, Pretorius D, Hull AD, Resnik R, Ramos GA. Identifying sonographic markers for placenta accreta in the first trimester. Journal of ultrasound in medicine : official journal of the American Institute of Ultrasound in Medicine. 2012 Nov;31(11):1835-41.

10. Ballas J, Pretorius DH, Resnik R, Ramos GA. OC20.06: Ultrasound findings of placenta accreta in the first trimester. Ultrasound Obstet Gynecol Ultrasound in Obstetrics \& Gynecology. 2011;38(S1):38.

11. Moretti FM, Fung K, Alali W, Oppenheimer L. P30.05: Diagnosis of placenta accreta in early pregnancy: case report and review of literature. UOG Ultrasound in Obstetrics \& Gynecology. 2010;36(S1):284.

12. Rahimi-Sharbaf F, Jamal A, Mesdaghinia E, Abedzadeh-Kalahroudi M, Niroomanesh S, et al. Ultrasound detection of placenta accreta in the first trimester of pregnancy. Iranian journal of reproductive medicine. 2014;12(6):421-426.

13. Radhouane A, Ines B, Khaled N. Diagnostic and decision-making difficulties: Placenta accreta at nine weeks' gestation. Asian Pacific Journal of Reproduction. 2016 3//;5(1):84-86.
14. Rac MW, Moschos E, Wells CE, McIntire DD, Happe SK, et al. 863: Low implantation on first trimester ultrasound and subsequent placenta previa and accreta. YMOB American Journal of Obstetrics and Gynecology: Supplement. 2016;214(1):S450-S450.

15. Rashbaum WK, Gates EJ, Jones J, Goldman B, Morris A, et al. Placenta accreta encountered during dilation and evacuation in the second trimester. Obstetrics and gynecology. 1995 May;85(5 Pt 1):701-3.

16. Jauniaux E, Collins S, Burton GJ. Placenta accreta spectrum: pathophysiology and evidence-based anatomy for prenatal ultrasound imaging. Am J Obstet Gynecol. 2018 Jan;218(1):75-87.

17. Rac M, Moschos E, Wells E, McIntire DD, Dashe JS, et al. OP24.06: Ultrasound (US) findings of placenta accreta in the first trimester. Ultrasound Obstet Gynecol Ultrasound in Obstetrics \& Gynecology. 2014;44(S1):139.

18. Yang JI, Kim HY, Kim HS, Ryu HS. Diagnosis in the first trimester of placenta accreta with previous Cesarean section. Ultrasound Obstet Gynecol. 2009 Jul;34(1):116-8. 